\title{
Collaborative Robots for Infrastructure Security Applications
}

\author{
Yi Guo ${ }^{1}$, Lynne E. Parker ${ }^{2}$, and Raj Madhavan ${ }^{3}$ \\ 1 Department of Electrical and Computer Engineering, Stevens Institute of \\ Technology, Hoboken, NJ 07030, USA, yguo1@stevens.edu, \\ http://www.ece.stevens-tech.edu/ yguo \\ 2 Department of Computer Science, University of Tennessee, Knoxville, TN 37996, \\ USA, parker@cs.utk.edu, http://www.cs.utk.edu/ parker \\ 3 Computational Sciences Division, Oak Ridge National Laboratory, Oak Ridge, \\ TN 37831, USA, and Guest Researcher at the Intelligent Systems Division, \\ National Institute of Standards \& Technology (NIST), 100 Bureau Drive, \\ Gaithersburg, MD 20899-8230, USA, raj.madhavan@ieee.org
}

We discuss techniques towards using collaborative robots for infrastructure security applications. A vast number of critical facilities, including power plants, military bases, water plants, air fields, and so forth, must be protected against unauthorized intruders. A team of mobile robots working cooperatively can alleviate human resources and improve effectiveness from human fatigue and boredom. This chapter addresses this scenario by first presenting distributed sensing algorithms for robot localization and 3D map building. We then describe a multi-robot motion planning algorithm according to a patrolling and threat response scenario. Neural network based methods are used for planning a complete coverage patrolling path. A block diagram of the system integration of sensing and planning is presented towards a successful proof of principle demonstration. Previous approaches to similar scenarios have been greatly limited by their reliance on global positioning systems, the need for the manual construction of facility maps, and the need for humans to plan and specify the individual robot paths for the mission. The proposed approaches overcome these limits and enable the systems to be deployed autonomously without modifications to the operating environment.

\subsection{Introduction}

The events of September 11, 2001 on United States soil have greatly increased the need to safeguard the country's infrastructure. A vast number of critical facilities need to be guarded from unauthorized entry. Unfortunately, the

Yi Guo et al.: Collaborative Robots for Infrastructure Security Applications, Studies in Computational Intelligence (SCI) 50, 185-200 (2007)

www.springerlink.com

(c) Springer-Verlag Berlin Heidelberg 2007 
number of security officials required to protect these facilities far exceeds their availability. Due to the enormity of this task, it seems unlikely that sufficient human resources can be committed to this infrastructure protection. An alternative approach is to allow technology to assist in this protection, through the use of multiple mobile robots capable of collaborating to guard the grounds of these important facilities from intrusion. Multi-robot systems can thus alleviate the onerous tasks faced by law enforcement officials and army personnel in surveillance, infrastructure security and monitoring of sensitive national security sites (e.g. nuclear facilities, power and chemical plants), building and parking lot security, warehouse guard duty, monitoring restricted access areas in airports and in a variety of military missions.

The Mobile Detection Assessment and Response System (MDARS) described in [7] was developed to provide an automated intrusion detection and inventory assessment capability for use in Department of Defense (DoD) warehouses and storage sites in United States. In this research, the operating area is previously mapped and the positions of the principal features of navigational interest are known in advance. The major sensory characteristics of these features are assumed to be known. By monitoring the variable features of the environment, an intrusion threat is detected. The system adopts random patrols in the secured area.

Another significant work in the area is described in [2], which details a robotic perimeter detection system where a cooperating team of six sentry vehicles are employed to monitor alarms. Formation of vehicles is achieved by teleoperation, while navigation of vehicles to a specified location is achieved by having robots use DGPS (Differential Global Positioning System) to follow specific paths defined by the human. These vehicles have also been used to remotely surround a specified facility. Mission planning is again achieved with the aid of an operator. An operator in the base station uses a graphic interface to determine paths for individual robots and develops a plan outlining obstacles and goal perimeters. The robots then execute this plan by following their designated paths. There are two important disadvantages of this approach from the perspectives of sensing and planning:

- The success of the mission is entirely dependent on positioning information provided by DGPS. Multipathing ${ }^{4}$ errors make it extremely difficult in many environments to obtain position estimates based on DGPS alone. Thus it becomes necessary to develop a scheme in which observations from relative and absolute sensors can be fused to continually deliver reliable and consistent position information for satisfying dynamic mission and motion planning requirements.

- Mission and path planning are fully dependent on the human operator and the system is incapable of dealing with dynamic situation changes that

\footnotetext{
${ }^{4}$ Multipathing refers to the situation where the signals detected by the DGPS receiver have been reflected off surfaces prior to detection instead of following the straight line path between the satellite and the receiver.
} 
require quick responses and mission and/or path replanning. Even if we did want to use a human operator to specify robot patrol routes, it will be quite difficult for a human to subdivide the patrol region amongst the robots to maximize efficiency. In this case, techniques are needed for dynamic multi-robot motion planning as an aid to the human for determining the best routes to provide to the robots.

There is little work on general frameworks or techniques developed for infrastructure security applications using collaborative robot teams. In this chapter, we formulate the research problems from an infrastructure security scenario, and report on new developments in distributed sensing and motion planning towards such important applications.

\subsection{Infrastructure Security Scenario and Research Problems}

We envision our new research advances to be used in an infrastructure security scenario such as the following. In an outdoor environment, robot teams are first sent out in a training phase to use our distributed sensing and positioning approach to build 3D digital elevation and obstacle maps of the area to be secured. Once the terrain is learned, the robots will be put into operation and each will operate in one of two modes:

1. a nominal patrol mode,

2. a threat response mode.

In general, robots will operate most often in the nominal patrol mode. In this mode, robots will use our new dynamic multi-robot motion planning algorithms to select efficient multi-robot patrol patterns. Each robot will then patrol its selected region. For efficient patrolling of each area, patrol paths need to be planned according to terrain features and local maps to achieve efficiency. While patrolling, the robots monitor their individual coverage areas for intrusion and also update their local terrain maps to account for environmental changes (e.g., changes in positions of authorized equipment, vehicles, etc.). If an intrusion is detected, some of the robots enter the threat response mode, as pre-defined by the rules of engagement set forth at the beginning of the team deployment. One example of a threat response would be for the detecting robot to send an alert to the human monitor (who is at a remote location), and then for a few robots to surround the threat and return video from multiple perspectives. To successfully respond to the threat, the robots need to dynamically plan paths to the threat location so that they reach the threat area in the shortest possible time. The remaining robots must subsequently replan their patrol paths to compensate for the robots that have entered the threat response mode.

The development of multi-robot teams for use in real world security applications in unstructured outdoor environments presents several challenging issues, which include the following three key research problems: 
1. distributed sensing for robot localization and 3D map building,

2. dynamic multi-robot motion planning,

3. integration of approaches to generate a proof of principle demonstration in a relevant infrastructure security environment.

Algorithms need to be developed for collaborative robots to operate in a reliable and robust manner and to be capable of operating in unstructured and dynamic environments with minimal modifications to the operating domain. In the following sections, we address each of these three research problems, presenting approaches and algorithms.

\subsection{Multi-Robot Positioning and Mapping using Distributed Sensing}

To accomplish missions in infrastructure security applications, multi-robot teams should be able to both autonomously position themselves and construct 3D elevation maps for efficient path planning when traversing on rugged uneven terrain. The objective is to design distributed sensing techniques and to develop schemes that ensure efficient utilization of sensor data obtained from sensors situated across the team members for multi-robot positioning and 3D elevation mapping. The sensors that are considered are: DGPS, wheel encoders, scanning laser rangefinders, inclinometers, compass and pan-tilt-zoom cameras.

\subsubsection{Heterogeneous Distributed Multi-Robot Localization}

To achieve real-time multi-robot cooperative positioning and mapping competency in a reliable and robust fashion, the sensing and the ensuing data fusion processes are of utmost importance. Thus, careful attention needs to be devoted to the manner in which the sensory information is integrated and interpreted. To satisfy this requirement, we propose a distributed multi-robot Extended Kalman Filter (EKF) estimation-theoretic scheme that enables efficient data fusion of sensor measurements from dead-reckoning and absolute sensors to continually deliver reliable and consistent pose (position and orientation) estimates. The robots collect sensor data regarding their own motion and share this information with the rest of the team during the EKF update cycles. The EKF processes the individual positioning information available from all the members of the team and produces a pose estimate for every one of them. Once pose estimates are available, a 3D map of the terrain can be generated by combining vision-based depth estimates with an elevation profile. The elevation profile may be obtained by fusing vertical displacements from DGPS with those computed from inclinometer pitch angles. The proposed scheme has several advantages. The uncertainty associated with measurements from different sensors is explicitly taken into account by using appropriate sensor models and validation procedures. It also becomes possible 


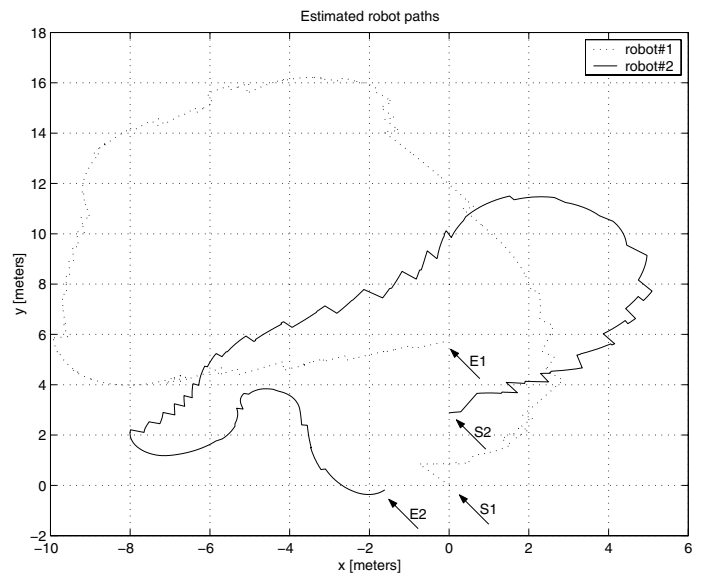

(a)
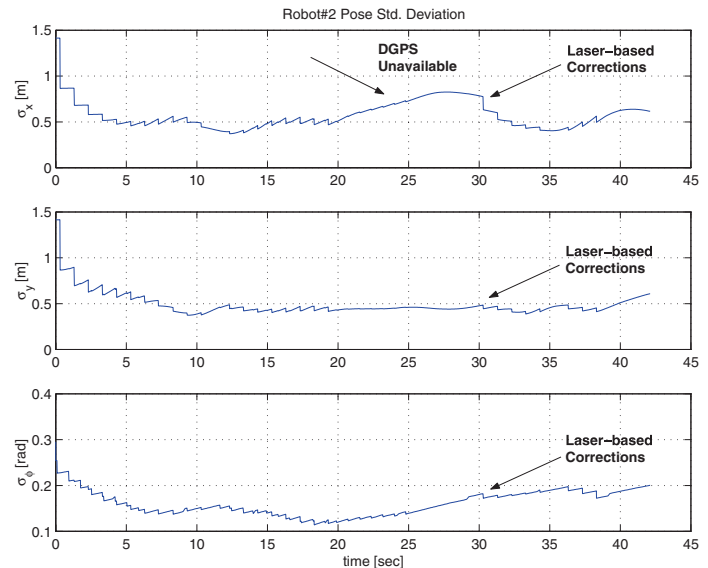

(b)

Fig. 9.1. The robots perform laser-based cooperative localization when DGPS becomes unavailable or when there are not enough satellites in view. EKF estimated robot paths are shown in (a). The solid line denotes the estimated path of robot \#2 and the dotted line that of robot \#1. (S1,E1) and $(\mathrm{S} 2, \mathrm{E} 2)$ denote the start and end positions for robots \#1 and \#2, respectively. The standard deviations of the pose of robot \#2 during laser-based cooperative localization are shown in (b). The external corrections offered by the laser-based localization scheme are marked by arrows. 
to combine measurements from a variety of different sensors as the estimation process is distributed across the robots.

When the quality of measurements from absolute sensors aboard the individual robots deteriorate ${ }^{5}$ or simply when a particular robot of a team does not have adequate sensing modalities at its disposal, another robot in the team with better sensing capability can then assist the deficient member(s) of the team such that the measurement from a single robot can be beneficial to the whole team. Thus, the heterogeneity of the team can be exploited to provide position estimates for all the team members [9].

Let us consider the case when the team is comprised of two robots. When robots \#1 and \#2 meet, they exchange relative pose information and the observation model becomes:

$$
\mathbf{z}_{c_{k}}=\left[\begin{array}{c}
x_{1_{k}}-x_{2_{k}} \\
y_{1_{k}}-y_{2_{k}} \\
\phi_{1_{k}}-\phi_{2_{k}}
\end{array}\right]+v_{12_{k}}=\mathbf{H}_{12_{k}} \mathbf{x}_{c_{k}}+v_{12_{k}}
$$

where $v_{12_{k}}$ refers to the uncertainty present in the relative pose observation and is modeled as a zero-mean uncorrelated Gaussian sequence with covariance $\mathbf{R}_{12_{k}}$.

The residual and the residual covariance are:

$$
\begin{aligned}
\nu_{c_{k}} & =\mathbf{z}_{c_{k}}-\hat{\mathbf{z}}_{c_{k}}=\mathbf{z}_{c_{k}}-\mathbf{H}_{12_{k}} \mathbf{x}_{c_{(k \mid k-1)}} \\
\mathbf{S}_{c_{k}} & =\mathbf{H}_{12_{k}} \mathbf{P}_{c_{(k \mid k-1)}} \mathbf{H}_{12_{k}}^{T}+\mathbf{R}_{12_{k}}
\end{aligned}
$$

The Kalman gain matrix, the state estimate and covariance updates (centralized) are as below:

$$
\begin{aligned}
\mathbf{W}_{c_{k}}= & \mathbf{P}_{c_{(k \mid k-1)}} \mathbf{H}_{12_{k}}^{T} \mathbf{S}_{c_{k}}^{-1} \\
\mathbf{x}_{c_{(k \mid k)}}= & \mathbf{x}_{c_{(k \mid k-1)}}+ \\
& \mathbf{W}_{c_{k}}\left[\mathbf{z}_{c_{k}}-\left(\mathbf{x}_{1_{(k \mid k-1)}}-\mathbf{x}_{2_{(k \mid k-1)}}\right)\right] \\
\mathbf{P}_{c_{(k \mid k)}}= & \mathbf{P}_{c_{(k \mid k-1)}}-\mathbf{W}_{c_{k}} \mathbf{S}_{c_{k}} \mathbf{W}_{c_{k}}^{T}
\end{aligned}
$$

where $\mathbf{x}_{c_{(k \mid k-1)}}$ and $\mathbf{P}_{c_{(k \mid k-1)}}$ are the state and covariance predictions, respectively.

Suppose that robot \#2 has a scanning laser rangefinder and also that the number of satellites in view from the current position of this robot indicates that DGPS is unavailable. (In the field trial, this corresponded to the robot going under a tree.) Given the pose of robot \#1 whose on-board sensors indicate a high level of confidence in their measurements, relative pose between robots \#2 and \#1 is determined as follows:

\footnotetext{
${ }^{5}$ The error in the DGPS positions can be obtained as a function of the number of satellites acquired and this error can then be used as an indicator of the deterioration of the quality of the sensor. As an alternative, the so-called dilution of precision measure associated with the GPS can be used for the same purpose.
} 
- Robot \#2 identifies robot \#1 and acquires a range and bearing laser scan.

- Robot \#1 communicates its pose to robot \#2.

- After necessary preprocessing to discard readings that are greater than a predefined threshold, the range and bearing to the minima identified in the laser profile of robot \#1 are determined.

- From the range and bearing pertaining to the minima, the pose of robot \#2 is then inferred.

- Since robot \#1 makes its pose available to robot \#2, relative pose information is obtained by comparing the two poses and is now available for use in Equation (9.1).

Within the EKF framework, state prediction takes place on individual robots in a decentralized and distributed fashion. By exchanging relative pose information, the states of the robots are then updated in a centralized fashion. The results for the laser-based cooperative localization are shown in Figures 9.1(a) and (b). Figure 9.1(a) shows the estimated paths of robots \#1 and \#2. The pose standard deviations of robot\#2 in Figure 9.1(b) demonstrate the utility of the relative pose information in accomplishing cooperative localization. At time $=21$ seconds, DGPS becomes unavailable as indicated by the rise in the $x$ standard deviation. It can be seen that as a result of the laserbased relative position information, there is a sharp decrease in the position standard deviations of robot \#2 (marked by arrows). As the motion of the robot is primarily in the $x$ direction when the corrections are provided, the resulting decrease in the $x$ standard deviation is noticeable compared to those in $y$ and $\phi$.

\subsubsection{Terrain Mapping}

Incremental terrain mapping takes place via four main processes:

- An incremental dense depth-from-camera-motion algorithm is used to obtain the depth to various features in the environment. The relative pose of the vehicles at these locations as well as depth covariances are associated with particular depth information. These covariances are used to determine regions which contain features of interest that should be indicated on the map.

- An elevation gradient of the terrain is determined by fusing GPS altitude information with vertical displacements obtained from inclinometer pitch angles. - The depth and elevation information are then registered with their associated covariances.

- The terrain map is updated to incorporate the registered values at their proper coordinates. The covariances associated with each measurement provide the confidence the algorithm has in that measurement. In the case of overlapping areas, this confidence determines whether or not the map is updated. 
An overall schematic diagram of the algorithm is given in [3]. Both the elevation profile for the motion segments and the feature locations are mapped, as shown in the partially updated terrain map (Figure 9.2). This Figure shows the elevation profile across the area traversed by each robot (in the locally fixed coordinate frame centered at the DGPS base station location) and prominent features within the robot's field of view during the motion segment are marked on the map.

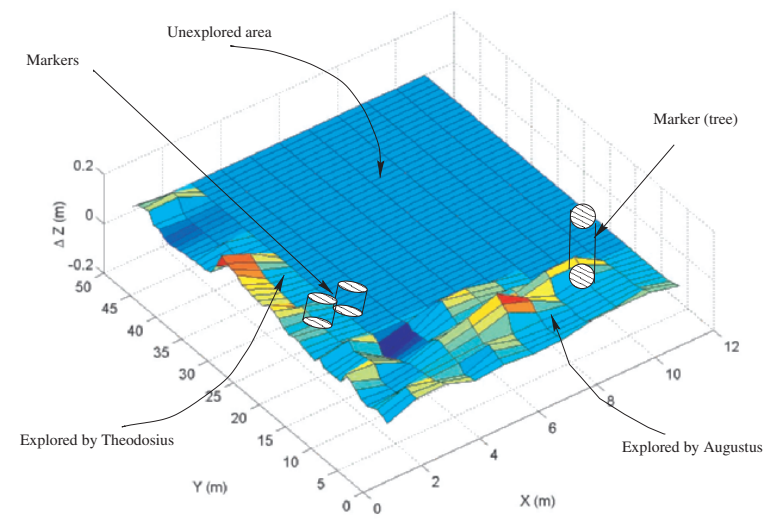

Fig. 9.2. Partially updated terrain map.

\subsection{Dynamic Multi-Robot Motion Planning}

According to the scenario presented in Section 9.2, the overall patrolling and threat response behavior can be divided into the following design modules:

1. Partition the patrolling region according to the number of robots;

2. Distribute robots from their initial positions to their sub-regions for patrolling;

3. Each robot patrols its sub-region continuously;

4. If a threat is detected by at least one robot during the patrol, a threat alert signal and the threat location are broadcast among robots. A subset of robots move from their current position to the threat position, and the rest of the team repeats steps 1 to 3 to provide continuous patrolling.

In the following, we describe autonomous region partitioning and motion planning in each of the above design modules. 


\subsubsection{Area Partition}

To achieve effective patrolling by a multi-robot team, the first task is to partition an area into sub-areas so that a utility function of the group is minimized. Mathematically, we formulate the problem as follows:

Consider a metric space $Q$, and $n$ robots with their positions at $\left\{p_{1}, p_{2}, \ldots, p_{n}\right\}$. For any point $q \in Q$, assume there is a cost function $f\left(q, p_{i}\right), i \in[1,2, \ldots, n]$ associated with it. If

$$
f\left(q, p_{i}\right)<f\left(q, p_{j}\right), i, j \in[1,2, \ldots, n], i \neq j
$$

we define the decentralized cost function:

$$
f_{i}\left(q, p_{i}\right)=f\left(q, p_{i}\right) .
$$

A group utility function is defined by

$$
U\left(p_{1}, p_{2}, \ldots, p_{n}\right)=\sum_{i=1}^{n} \int_{Q} f_{i}\left(q, p_{i}\right) d q
$$

The objective is to find solution $\left(p_{1}, p_{2}, \ldots, p_{n}\right)$ so that the group utility function $U$ is minimized.

We know that the set $\left(p_{1}, p_{2}, \ldots, p_{n}\right)$ satisfying

$$
\frac{\partial U\left(p_{1}, p_{2}, \ldots, p_{n}\right)}{\partial p_{i}}=0
$$

is the solution for $\min U(\cdot)$.

In a special case when $Q$ is a finite dimensional Euclidean space, and the cost function $f\left(q, p_{i}\right)$ is chosen to be the distance between $q$ and $p_{i}$, i.e., $f\left(q, p_{i}\right)=\operatorname{dist}\left(q, p_{i}\right)$, the set of points satisfying (9.2) compose Voronoi region $V_{i}=V_{i}\left(p_{i}\right)$. The set of regions $\left\{V_{1}, V_{2}, \ldots, V_{n}\right\}$ is called the Voronoi diagram for the generators $\left\{p_{1}, p_{2}, \ldots, p_{n}\right\}$. In this case, the solution to (9.4) is the mass centroid of $V_{i}$. Centroidal Voronoi tessellations [1] provides solution methods to find the mass centroids.

Applying the above theoretic results to the area partition, we generate the Voronoi diagram and the mass centroids $p_{i}, i \in[1,2, \ldots, n]$, which are the closest point to every point in the Voronoi region $V_{i}$.

\subsubsection{Initial Distribution}

After the set of points $\left\{p_{1}, p_{2}, \ldots, p_{n}\right\}$ are generated, we need to move the $i$ th robot from its initial position to $p_{i}$, so that the robot can patrol the Voronoi region $V_{i}$. The motion planning problem for this sub-task is defined as follows:

Find feasible trajectories for the robot, enrouting from its initial position to its goal position $p_{i}$, without collisions with static and dynamic obstacles in the environment while satisfying nonholonomic kinematics constraints.

Solution methods can be found in $[6,12]$. 


\subsubsection{Complete Coverage Patrolling}

In this sub-task, each robot needs to plan its patrolling path in its own Voronoi region $V_{i}$. Multiple paths can be planned based on different criterion, for example, complete coverage of the area as high a frequency as possible, maximize area covered in unit time, minimize repeat coverage, etc. For complete coverage, the robot patrols the region so that every point in the region is covered within the robot sensor range at least once over a time period.

We propose a path planning algorithm for complete region coverage. It first packs the bounded region with disks of radius $R_{c}$. It was shown in [5] that the disk placement pattern in Figure 9.3 has a minimum number of disks to cover a rectangle. Since the radius of the disk is the same as the coverage range of robot's sensors, complete coverage with minimum repeated coverage can be achieved by visiting every center of the disks. Complete coverage path planning is then to find the sequence to visit the centers. A path planning algorithm was proposed to find a complete coverage path in [5]. However, the algorithm works only in environments without obstacles. Neural network models were used for robot motion planning in dynamic environments in [10] since the dynamically varying environment can be represented by the dynamic neural activity generated by the model. In [13], a neural network approach was developed for complete coverage path planning in a nonstationary environment. We modify the algorithm corresponding to our data structure and generate collision-free complete coverage paths.

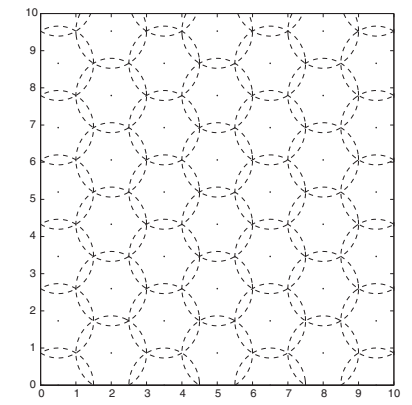

Fig. 9.3. Covering a rectangle using a minimum number of disks

The basic idea of the neural network approach is to generate a dynamic landscape for the neural activities, such that through neural activity propagation, the uncleaned areas $^{6}$ globally attract the robot in the entire state space, and the obstacles locally repel the robot to avoid collisions. The dynamics of each neuron in the topologically organized neural network is characterized by a shunting equation derived from Hodgkin and Huxley's membrane equation

\footnotetext{
${ }^{6}$ The uncleaned areas are defined as the uncovered disks, and the obstacle areas are defined as the disks that are occupied by obstacles.
} 
[8]. The robot path is autonomously generated from the activity landscape of the neural network and the previous location. The neural network model is expressed topologically in a discretized workspace. The location of the neuron in the state space of the neural network uniquely represents an area. In the proposed model, the excitatory input results from the unclean areas and the lateral neural connections, whereas the inhibitory input results from the obstacles only. The dynamics of the neuron in the neural network is characterized by $(9.5)$ :

$$
\begin{aligned}
\dot{x}_{i}= & -A x_{i}+\left(B-x_{i}\right)\left(\left[I_{i}\right]^{+}+\sum_{j=1}^{k} w_{i j}\left[x_{j}\right]^{+}\right) \\
& -\left(D+x_{i}\right)\left[I_{i}\right]^{-}
\end{aligned}
$$

where $k$ is the number of neural connections of the $i$ th neuron to its neighboring neurons within the receptive field. Six neighbors of a point (neuron) are shown in Figure 9.4.

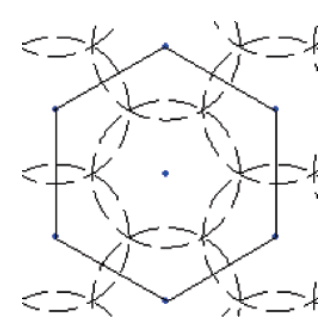

Fig. 9.4. Six neighbors of a neuron

The external input $I_{i}$ to the $i$ th neuron is defined as in (9.6).

$$
I_{i}= \begin{cases}E & \text { if it is an unclean area } \\ -E & \text { if it is an obstacle area } \\ 0 & \text { otherwise }\end{cases}
$$

where $E>>B$ is a very large positive constant. The terms $\left[I_{i}\right]^{+}+\sum_{j=1}^{k} w_{i j}\left[x_{j}\right]^{+}$ and $\left[I_{i}\right]^{-}$are the excitatory and inhibitory inputs respectively. Function $[a]^{+}$ is a linear threshold function defined as $[a]^{+}=\max \{a, 0\}$, and $[a]^{-}=$ $\max \{-a, 0\}$. The connection weight $w_{i j}$ between the $i$ th and the $j$ th neurons is 1 if they are neighbors or 0 if they are not neighbors.

To make the path having less navigation turns, for a current robot location $p_{c}$, we select the next point $p_{n}$ within the uncleaned neighbors according to $(9.7)$ :

$$
x_{n}=\max \left\{x_{j}+\left(1-\frac{\Delta \theta_{j}}{\pi}\right), j=1,2, \ldots, k\right\}
$$

where $k$ is the number of neighboring neurons, $x_{j}$ is the neuron activity of the $j$ th neuron, $\Delta \theta_{j}$ is the absolute angle change between the current and 
next moving directions, i.e., $\Delta \theta_{j}=0$ if going straight, and $\Delta \theta_{j}=\pi$ if going backward. After the robot reaches its next position, the next position becomes a new current position. Because of the excitatory neural connections in (9.5), the neural activity propagates to the entire state space so that the complete coverage is achieved.

A complete coverage path in an environment with stationary obstacles is shown in Figure 9.5. It is shown that the path completely covers the bounded region without covering a point twice. Note that in a trap situation, that is, there are no uncleaned neighbors, the neighbor's neighbors become the neighbors of the neuron, so that uncleaned area can be continuously searched. This can be seen from the right bottom and top middle parts of the figure. The algorithm works for moving obstacles if the speed of the obstacles are known, since the landscape activities of the environment are updated dynamically in the algorithm.

Cooperative patrolling paths of four robots are shown in Figure 9.6.

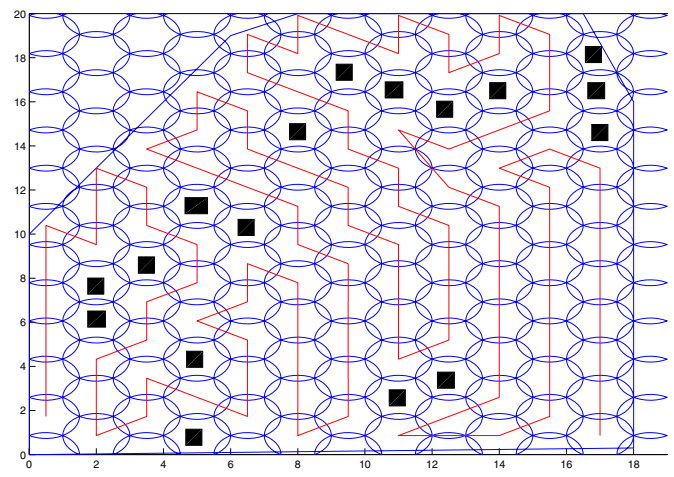

Fig. 9.5. Complete coverage paths, the dark rectangles are stationary obstacles

\subsubsection{Point Convergence}

In this module, a subset of robots move to the threat location from their current positions. The motion planning problem for each robot becomes: given a start and a goal, generate a feasible trajectory without collisions. The same solution methods as described in subsection 9.4.2 can be applied. 


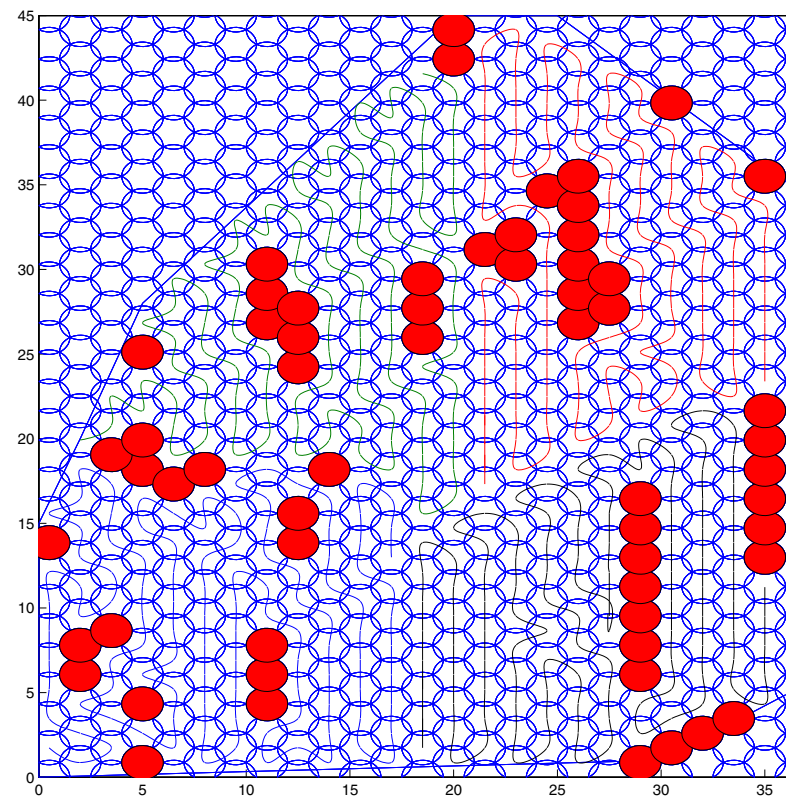

Fig. 9.6. Cooperative coverage trajectories: each continuous curve represents one robot's trajectory, and the solid circles denote areas occupied by stationary obstacles.

\subsection{System Integration Towards Proof of Principle Demonstration}

For a successful proof of principle demonstration in a relevant infrastructure security environment, functionalities of distributed sensing developed in Section 9.3 should be integrated with the dynamic motion planning capabilities in Section 9.4 to realize the cooperative team objectives. Figure 9.7 illustrates the block diagram of system integration. Note that in the figure, techniques regarding threat detection (in the gray box) are not discussed in this chapter.

We have partially implemented the algorithms proposed on a group of ATRV-mini robots. The experimental setup is shown in Figure 9.8. It consists of a wireless mini-LAN, a Local Area DGPS (LADGPS), a software platform (Mobility from RWI) and codes developed in-house under Linux to read and $\log$ the data for the sensors on each robot. The wireless LAN is set up outdoors between an Operator Console Unit (OCU) and the robots. The OCU consists of a rugged notebook equipped with a BreezeCOM access point and antenna. Each robot has a BreezeCOM station adaptor and an antenna. The LADGPS is formed by the base station/antenna hardware connected to the OCU and 


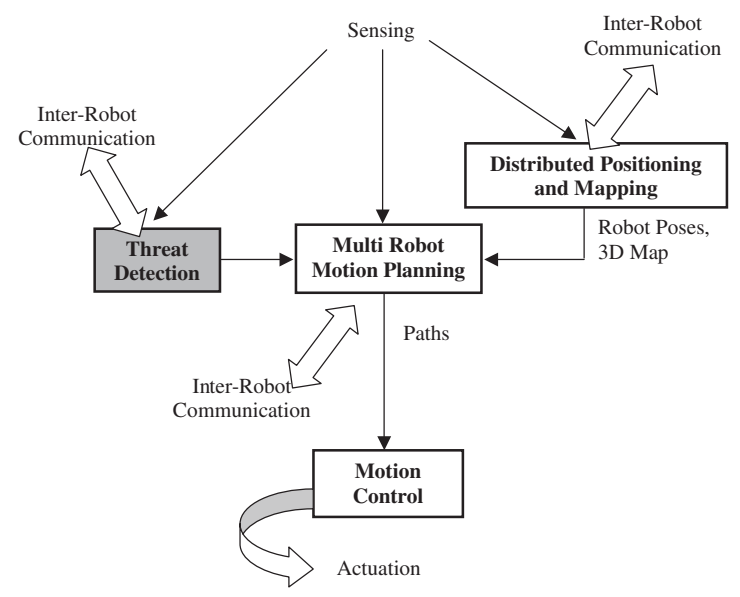

Fig. 9.7. Block diagram of system integration.

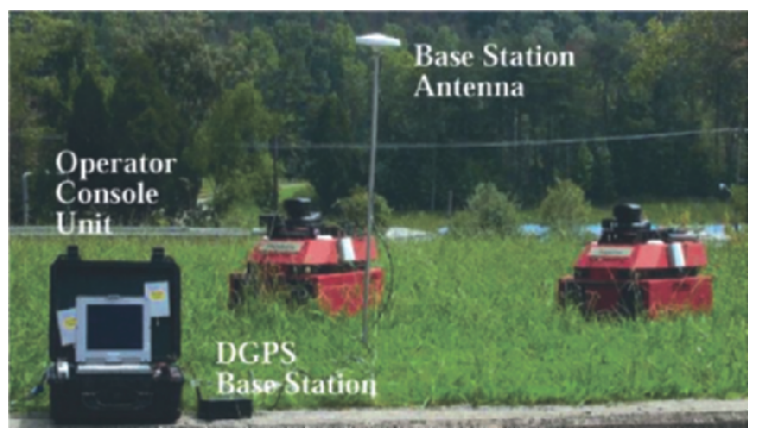

Fig. 9.8. Experimental setup in an outdoor environment

remote stations/antennas directly mounted on each robot. Each robot's station receives differential corrections from the base station such that LADGPS accuracy of up to 10 centimeters is obtainable. Some experimental results can be found in our previous publications $[3,4,9,11]$.

\subsection{Conclusions}

Recent terrorist events on United States soil have dramatically increased the need for protection of our nation's infrastructure. Rather than stretch alreadythin human resources to guard facilities against low-probability intrusions, technological solutions are preferred. We propose to address this problem by using teams of intelligent robots for infrastructure security applications.

We first formulate the research problems from an infrastructure scenario, and then propose new algorithms in distributed sensing and multi-robot 
motion planning to achieve the autonomous patrolling and threat response tasks. Finally, the system integration of sensing and planning are presented towards a successful proof of principle demonstration. The developed collaborative sensing and motion control strategies enable a robot team to position themselves and move appropriately in a previously unknown environment to enable intrusion detection. To briefly summarize the advances of our approach over existing approaches for infrastructure security in outdoor environments, we compare different approaches in Table 9.1. Future work includes extensive real robot experiments using the experimental setup in Section 9.5.

Table 9.1. Comparison of approaches

\begin{tabular}{l|c|c|c}
\hline & $\begin{array}{c}\text { Proposed } \\
\text { Approach }\end{array}$ & $\begin{array}{c}\text { Approach } \\
\text { in }[2]\end{array}$ & $\begin{array}{c}\text { Approach } \\
\text { in }[7]\end{array}$ \\
\hline Autonomous detection of threats & $\mathrm{X}$ & $\mathrm{X}$ & $\mathrm{X}$ \\
\hline Mobility for rapid response & $\mathrm{X}$ & $\mathrm{X}$ & $\mathrm{X}$ \\
\hline No infrastructure modifications required & $\mathrm{X}$ & $\mathrm{X}$ & \\
\hline No dependency on absolute positioning (DGPS) & $\mathrm{X}$ & & \\
\hline Autonomous path planning & $\mathrm{X}$ & & \\
\hline No a priori map needed & $\mathrm{X}$ & & \\
\hline
\end{tabular}

\section{References}

1. Q. Du, V. Faber, and M. Gunzburger. Centroidal Voronoi tessellations, theory, algorithms and applications. SIAM Review, 41:637-676, 1999.

2. J. T. Feddema, C. Lewis, and P. Klarer. Control of multiple robotic sentry vehicles. In Proceedings of SPIE Unmanned Ground Vehicle Technology, SPIE, volume 3693, pp. 212-223, 1999.

3. K. Fregene, R. Madhavan, and L.E. Parker. Incremental Multiagent Robotic Mapping of Outdoor Terrains. In Proceedings of the IEEE International Conference on Robotics and Automation, pp. 1339-1346, May 2002.

4. Y. Guo and L. E. Parker. A distributed and optimal motion planning approach for multiple mobile robots. In Proceedings of IEEE International Conference on Robotics and Automation, pp. 2612-2619, May 2002.

5. Y. Guo and Z. Qu. Coverage control for a mobile robot patrolling a dynamic and uncertain environment. In Proceedings of World Congress on Intelligent Control and Automation, pp. 4899-4903, China, June 2004.

6. Y. Guo, Z. Qu, and J. Wang. A new performance-based motion planner for nonholonomic mobile robots. In Proceedings of the 3rd Performance Metrics for Intelligent Systems Workshop, NIST, Gaithersburg, MD, Sept. 2003.

7. T. Heath-Pastore, H.R. Everett, and K. Bonner. Mobile Robots for Outdoor Security Applications. In Proceedings of the American Nuclear Society 8th International Topical Meeting on Robotics and Remote Systems, April 1999. 
8. A. L. Hodgkin and A. F. Huxley. A quantitative description of membrane current and its application to conduction and excitation in nerve. J. Physiol. Lond., 117:500-544, 1952.

9. R. Madhavan, K. Fregene, and L.E. Parker. Distributed Cooperative Outdoor Multirobot Localization and Mapping. In Autonomous Robots (Special Issue on Analysis and Experiments in Distributed Multi-Robot Systems), Vol. 17, Issue 1, pp. 2339, 2004.

10. F. Muniz, E. Zalama, P. Gaudiano, and J. Lopez-Coronado. Neural controller for a mobile robot in a nonstationary environment. In Proceedings of $2 n d$ IFAC Conference on Intelligent Autonomous Vehicles, Helsinki, Finland, pp. 279-284, 1995.

11. L. E. Parker, K. Fregene, Y. Guo, and R. Madhavan. Distributed heterogeneous sensing for outdoor multi-robot localization, mapping, and path planning. In A. C. Schultz and L. E. Parker, editors, Multi-Robot Systems: From Swarms to Intelligent Automata, pp. 21-30. Kluwer, The Netherlands, 2002.

12. Z. Qu, J. Wang, and C. E. Plaisted. A new analytical solution to mobile robot trajectory generation in the presence of moving obstacles. In Proceedings of 2003 Florida Conference on Recent Advances in Robotics, May 2003. Also submitted to IEEE Transactions on Robotics and Automation.

13. S. X. Yang and C. Luo. A neural network approach to complete coverage path planning. IEEE Transactions on Systems, Man and Cybernetics - Part B: Cybernetics, 34(1):718-725, 2004. 